\title{
Fine-temporal forecasting of outbreak probability and severity: Ross River virus in Western Australia
}

\author{
I. S. KOOLHOF ${ }^{1 *}$, S. BETTIOL ${ }^{2}$ AND S. CARVER ${ }^{1 *}$ \\ ${ }^{1}$ School of Biological Sciences, University of Tasmania, Hobart, Tasmania, Australia \\ ${ }^{2}$ School of Medicine, University of Tasmania, Hobart, Tasmania, Australia
}

Received 5 April 2017; Final revision 18 June 2017; Accepted 2 August 2017; first published online 4 September 2017

\section{SUMMARY}

Health warnings of mosquito-borne disease risk require forecasts that are accurate at finetemporal resolutions (weekly scales); however, most forecasting is coarse (monthly). We use environmental and Ross River virus (RRV) surveillance to predict weekly outbreak probabilities and incidence spanning tropical, semi-arid, and Mediterranean regions of Western Australia (1991-2014). Hurdle and linear models were used to predict outbreak probabilities and incidence respectively, using time-lagged environmental variables. Forecast accuracy was assessed by model fit and cross-validation. Residual RRV notification data were also examined against mitigation expenditure for one site, Mandurah 2007-2014. Models were predictive of RRV activity, except at one site (Capel). Minimum temperature was an important predictor of RRV outbreaks and incidence at all predicted sites. Precipitation was more likely to cause outbreaks and greater incidence among tropical and semi-arid sites. While variable, mitigation expenditure coincided positively with increased RRV incidence $\left(r^{2}=0 \cdot 21\right)$. Our research demonstrates capacity to accurately predict mosquito-borne disease outbreaks and incidence at fine-temporal resolutions. We apply our findings, developing a user-friendly tool enabling managers to easily adopt this research to forecast region-specific RRV outbreaks and incidence. Approaches here may be of value to fine-scale forecasting of RRV in other areas of Australia, and other mosquito-borne diseases.

Key words: Arboviruses, Early warning forecasting, Epidemiology, Mosquito-borne disease.

\section{INTRODUCTION}

Arboviral diseases cause a significant impact upon human health worldwide, with multiple climatic zones (including tropical, semi-arid, and Mediterranean regions) experiencing increased burdens from a plethora of emerging and re-surging forms $[1,2]$. Owing to

\footnotetext{
* Authors for correspondence: I. S. Koolhof and S. Carver, School of Biological Sciences, University of Tasmania, Hobart, Tasmania, Australia and School of Medicine, University of Tasmania, Hobart, Tasmania, Australia.

(Email: koolhofi@utas.edu.au and Scott.Carver@utas.edu.au)
}

dependence on environmental features, their dynamics (particularly mosquito-borne pathogens) are often uniquely suited for forecasting using readily available environmental data. Because of this, the timing of disease control can be matched effectively with vectorial and disease risk. However, for many mosquito-borne diseases forecast modelling of risk is often at a coarse temporal resolution, such as monthly scales [3-5]. While this helps to understand disease dynamics, forecasts made on coarse temporal resolutions are often limited in their applicability to 'on the ground' disease management, such as though vector reduction and well- 
timed public awareness campaigns. To enhance the impact of disease control strategies, models that forecast disease at temporal scales relevant to management (e.g. weekly) and are also easily accessible to managers are highly valuable.

Epidemiologically Ross River virus (RRV), family Togaviridae genus Alphavirus, is Australia's most important vector-borne disease with 1451-9551 clinical notifications per year (per capita rate $>40 / 100$ $000)$ at an estimated annual health care and lost productivity cost of $\$ 15$ million [6-8]. The epidemiology of $\mathrm{RRV}$, like many mosquito-borne diseases, is fraught with complexity. The drivers involved in transmission span environmental conditions, vector and reservoir host dynamics, and existing human mitigation, all which vary across geographic and climatic regions $[9,10]$. RRV epidemic areas of Australia occur across tropical, semi-arid, Mediterranean, and temperate climates. Thus, the processes underpinning RRV epidemics and their severity are complex [11-13]. Despite this complexity, studies have shown that disease forecasts can be achieved moderately well using environmental drivers in many instances, as this can be representative of many aspects of the biological mechanisms determining transmission.

A multitude of studies incorporating various designs have been used over the past three decades to define environmental determinants of RRV notifications [e.g. 5, 14, 15], and indeed many important mosquitoborne diseases. A common theme among these studies is the importance of precipitation, temperature, tidal or flood variation, and humidity. These environmental determinants directly or indirectly shape mechanisms underscoring disease dynamics, including vector mosquito activity, reservoir hosts movement, breeding interactions, and viral activity [16]. For instance, the inundation of wetlands from precipitation and high tides (where vectors are halotolerant) may cause the movement of reservoir hosts from these areas closer to the boundaries of urban areas while also providing an ideal breeding site for the mosquitos [10, 16, 17]. The outcome of these environmental drivers can enhance vector-host interactions, viral transmission and spillover to humans [11, 18, 19]. Vectors in particular are highly responsive to changes in environmental conditions, having the greatest potential for rapid translation into increased abundance and infections [15, 20, 21].

While environmental factors can predict RRV notifications, it should be acknowledged that predicting RRV disease is best achieved using detailed knowledge of vector abundance [13] and, where available, host information [5]. However, collection of such detailed vector-host information is for many areas infeasible, particularly over long durations. In contrast, environmental information is often much more readily available and modelling approaches that can best exploit widely available data sources to predict and understand diseases are of significant value. However, not all environmental determinants are equal in all climates, and thus an appreciation of their relative importance for predicting RRV across geographic and climatic gradients is of value. Further, while previous forecast modelling has aided in the epidemiological understanding of RRV, very few provide an accessible format by which health departments may easily adopt modelling approaches to public health and disease reduction.

Here we tackle the essential challenge of predicting RRV at fine-temporal scales across multiple epidemic centres using environmental determinants of disease; and we produce a user friendly early warning system based on these findings. We use RRV notification data and environmental data over 23 years in five epidemiologically important centres in Western Australia, spanning tropical, semi-arid, and Mediterranean climates. We construct predictive models for RRV outbreaks and their severity/incidence (the probability of notifications above long-term averages and the number of disease notifications, respectively), and for one site, where data are available we determine how well timing of expenditure on mitigation coincides with RRV incidence. At a broad scale, this research fills the long-standing void in improving our understanding of environmental drivers on a finetemporal scale to forecast RRV transmission. Building from previous literature, we provide insight into the distinct differences between Mediterranean, semi-arid and tropical areas for RRV transmission. Knowledge of the fundamental differences in RRV predictors between these climatic regions is critical for environmental and public health managers when considering region-specific approaches to vector mitigation and public education programmes. Furthermore, we provide a means to improve predictive capabilities for RRV outbreaks where mosquito surveillance is not currently being conducted. We apply this research to create a userfriendly predictive tool meant to assist public health managers with timing of vector control, decision making, and public health disease risk awareness campaigns. 


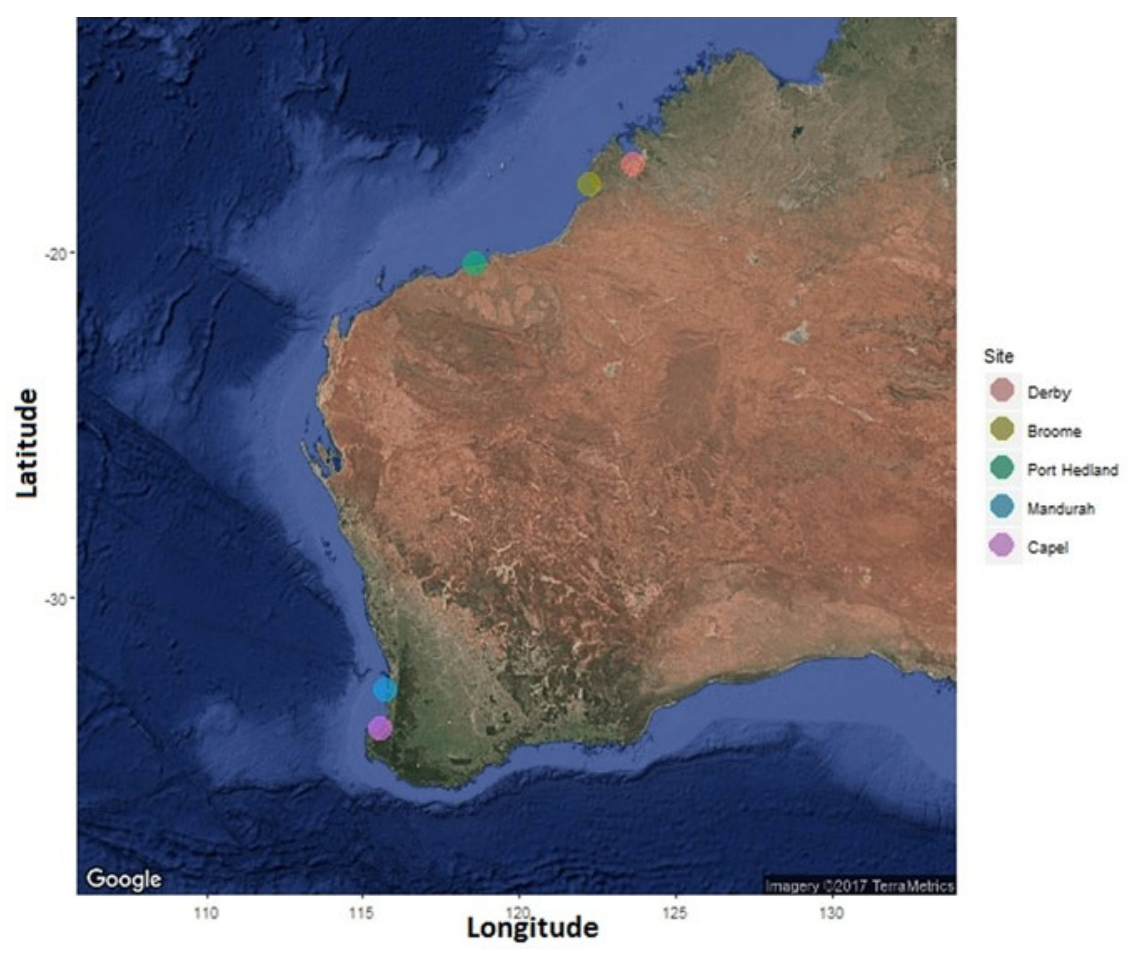

Fig. 1 Study sites. Map location of study sites. Showing Derby and Broome from a tropical region, Port Hedland from a semi-arid region, and Mandurah and Capel from a Mediterranean region.

\section{METHODS}

\section{Study sites}

Five sites in Western Australia were selected due to their high mosquito-borne disease attack rates and being epidemiologically important outbreak areas (Fig. 1) [6, 22-24]. The northern sites of Derby and Broome are situated in the Kimberly region and experience tropical monsoonal rainfall. Port Hedland, south of these two sites is in the Pilbara region which experiences a semi-arid climate. These northern sites commonly have Culex annulirostris (a freshwater breeding mosquito) and Aedes vigilax (a saltmarsh breeding mosquito) implicated in RRV transmission [10]. All northern sites have salt marshes neighbouring their town or shire. The southern sites of Mandurah and Capel are in the Peel and southwest geographic regions, respectively, a Mediterranean climatic area. At these sites two saltmarsh breeding mosquitoes, Aedes camptorhynchus and Ae. vigilax, are the primary vectors for RRV transmission [25]. Mandurah also plays host to one of the biggest estuarine systems in Southern Western Australia, with a multitude of canals and river systems stretching inland. All sites have mosquito vector dynamics locally driven by both tides and precipitation, except one site, the Shire of Capel, which is non-tidal and was selected to contrast the difference in drivers in RRV transmission for non-tidal and tidal sites of the Southern Mediterranean region.

\section{Data sources}

Data of RRV notifications was provided by the Western Australian Department of Health and the Western Australian Notifiable Infectious Diseases Database for the period of January 1991-December 2014 containing the number of notifications and the week of notification. Region-specific annual population data were collected from current and historical records from the Australian Bureau of Statistics [26]. $\mathrm{RRV}$ incidence for each region was then calculated using the respective annual population size with the number of RRV notifications per week to create incidence (per 1000 individuals) per week. A fixed incidence threshold was used as the definition of an outbreak, where RRV incidence above the long term average of the mean for each individual site resulted in an outbreak, excluding weeks where incidence were equal to zero [27]. Environmental data were collected for the entire study period and gaps in missing data recorded (Supplementary Table S1). 
Daily tidal data were collected from the Western Australian Department of Transport and the Australia Bureau of Meteorology for all sites excluding Capel (which does not have a locally occurring tidally driven saltmarsh). Tide data were then summarised into the recorded observed maximum and minimum weekly tide heights. Derby experiences very high tides, presenting some gauging problems, where the minimum tide measurement could only be recorded down to $375 \mathrm{~cm}$ (above sea level) where the tide gauge is placed. As tidal measure below $375 \mathrm{~cm}$ were not recorded in Derby, weekly minimum tide was omitted from the Derby analysis. Furthermore, it was rare for tidal data to be continuous for the complete period (1991-2014) for any site. Where this occurred, data from additional sites that were spatially close to our primary study areas were compared using cross-correlations to account for any time lags and then linear regression models used for interpolating missing values. Spatially matched sites included: Derby matched with Broome (from 1991 to 1995), and Port Hedland matched with Cape Lambert (years 1995, 1997, and 2013-2014). All tidal data were standardised to account for any alterations in instrument measurements. In Eqn. (1), $P_{i}$ is the adjusted environmental observation where values were missing, $\mu$ represents the observed environmental variable in the comparative data set, $\beta_{1}$ is the regression slope, and $\beta_{0}$ is the regression intercept.

$P_{i}=\mu \times \beta_{1}+\beta_{0}$.

Precipitation data were collected from the Bureau of Meteorology containing the amount of precipitation on a daily scale. The daily precipitation was summarised into weekly total precipitation. For sites which did not have complete continuous data, a relatively close comparative site was chosen, cross correlations and linear regressions were again used on to make interpolations, using the same methodology as described for tides. Comparative matched sites included: Derby matched with Yeeda (from 1991 to 1994), Mandurah matched with Karnet (from 2001 to 2014), and Capel matched with Thirlmere (from 2003 to 2014). All precipitation measurements are reported in millimetres.

Daily maximum and minimum temperatures $\left({ }^{\circ} \mathrm{C}\right)$ were also provided by the Australia Bureau of Meteorology with temperature being on a daily scale. Temperatures were summarised into weekly maximum and minimum mean temperatures. For non-continuous data, comparative sites were used, as described above. Comparative matched sites included: Derby matched with a station at the Royal Australian Air Force Base 'Curtin' (from 1991 to 1995), and Mandurah matched with Karnet (from 2001 to 2014). The Bureau of Meteorology has no temperature monitoring stations located in and around Capel. Temperature data for Capel were instead collected from the Shire of Donnybrook $24 \mathrm{~km}$ East.

\section{Statistical analyses}

Environmental predictors of $R R V$

We utilised a structured approach to link environmental predictors with RRV notifications. Firstly, we determined the optimal time-lags between environmental predictors and RRV incidence. To determine time lags between environmental variables and weekly RRV incidence we utilised cross-correlation analysis, a lagged effect was introduced for each environmental variable at each site $[28,29]$. Temporal lags account for effects of environmental predictors on the lifecycle of the virus and mosquitoes, human incubation prior to disease notification, and host dynamics $[5,16,30]$. Cut-off points for maximum lag periods included biological considerations, visual observation of relative peaks in RRV incidence in relation to the environmental variable, and relative comparisons with other lags presented in the literature [5, 29, 31]. This meant that the cut-off periods for each environmental variable were equal across all the sites. The given cutoff time periods included 5 weeks for tidal heights, 10 weeks for precipitation, and 15 weeks for temperature measurements. Before modelling, environmental variables were checked for multi-collinearity to ensure they were not correlated with one another $(>0.85$ Pearson's correlation; Supplementary Tables S2-S6).

Secondly, we applied two modelling approaches to link time-lagged environmental factors with weekly RRV: hurdle models (with a binomial logit function) for the probability of an outbreak and linear models for the severity (RRV incidence) of an outbreak. All predictor variable combinations were undertaken for each modelling approach and Akaike's Information Criterion correction corrected for sample sizes (AICc) were determined [32]. Rank of models was assessed by delta AICc [32].

Model-averaged coefficients of the model combinations were constructed, averaged variable estimates were derived from each model were the variable was included (see the 'Results' section). The estimates 
from the hurdle models were then back-transformed, exponentiating the model coefficients and used as predictors in forecasting the probability of a RRV outbreak (Eqn. (2)), where the severity of an outbreak (in incidence) was calculated based on the model-averaged coefficients from the linear regression models (Eqn. (3)). Where $Y_{i}$ represents your predicted outcome odd ratio and incidence (Eqns (2) and (3), respectively), $\beta_{0}$ represents the regression intercept, and $\beta_{1,2, p} x_{1,2, p_{i}}$ represents the beta coefficients from the regression models.

$\left(Y_{i}\right)=\left(\begin{array}{c}\exp +\left(\beta_{0}+\left(\beta_{1} x_{1_{i}}+\beta_{2} x_{2_{i}}+\cdots+\beta_{p} x_{p_{i}}\right)\right) \\ 1+\exp +\left(\beta_{0}+\left(\beta_{1} x_{1_{i}}+\beta_{2} x_{2_{i}}+\cdots+\beta_{p} x_{p_{i}}\right)\right)\end{array}\right)$,

$\left(Y_{i}\right)=\beta_{0}+\left(\beta_{1} x_{1_{i}}+\beta_{2} x_{2_{i}}+\cdots+\beta_{p} x_{p_{i}}\right)$.

In all cases, variable importance weights were also calculated representing the sum of the Akaike weights over all the models in which the variable appears [32].

The fit of models to the data were assessed using a variety of techniques. For outbreak probability, we calculated sensitivity, specificity, and negative and positive predicted values based on the model-averaged coefficients. Cross-validation was also conducted using a $K$-fold validation technique with the data being split into ten equal folds and the model being tested giving both a prediction error for the leave-one-out crossvalidation and an adjusted design to compensate for the leave-one-out cross-validation [33, 34]. The $K$-fold cross-validation prediction error is used to determine the amount of variation in the hurdle model predictions where we are testing predictions against the entire data available [35]. $K$-fold cross-validations were run ten times to obtain a standardised error to correct for possible errors produced in the data petitioning process. For outbreak severity we assessed model fit via $K$-fold cross-validations, again based on model-averaged coefficient effects.

\section{Relationship between $R R V$ incidence and expenditure}

Following modelling of RRV incidence based on environmental factors, we examined the relationship between timing of expenditure on RRV control and RRV incidence. This was undertaken for Mandurah, where monthly expenditure data were available from 2007 to 2012. Data were aggregated on a monthly basis. Monthly opposed to weekly data were utilised to account for infrequent mosquito control activity throughout each year. The model-averaged coefficients of RRV incidence in Mandurah were used to predict RRV incidence using a linear regression model (Supplementary Table S7). The residuals of the predicted incidence to predict true incidence were then used as the outcome variable predicted by monthly expenditure. To examine model robustness, a cross-validation of the linear regression was conducted. A 4-fold cross-validation was used due to the limited size of the data. Data partitioning were repeated ten times, allowing for equal amounts of observation in each partition for each model construction and comparison.

All statistical analyses described were performed in $\mathrm{R}$ (Version 3.2.5, www.r.project.org) using the packages 'pscl', 'MuMln', 'pROC', and 'boot' in RStudio (Version 0.99.489).

Using the modelled average coefficients from predicting RRV outbreaks (Eqn. (2)) and incidence (Eqn. (3)), we constructing a user friendly Microsoft Excel-based tool capable of predicting a minimum of 1 week into the future for each site studied (Supplementary Table S8). All supplementary materials are available on the Cambridge Core website.

\section{RESULTS}

There were 3567 notified cases of RRV across all sites for the study period; these included Derby with 134, Broome with 635, Port Hedland with 349, Mandurah with 2167, and Capel with 282 notified cases. The various numbers of cases across the sites predominantly reflect the population differences between the sites.

\section{The weekly probability of an outbreak}

An outbreak was defined by the mean weekly incidence (weeks of zero incidence were omitted), whereby values above the mean were classified as an outbreak for each site. The incidence (per/1000 individuals) which characterised an outbreak were 0.165 in Derby, 0.169 in Broome, 0.128 in Port Hedland, 0.055 in Mandurah, and 0.244 in Capel and represented as a threshold in Figure 2. The occurrence of outbreaks differed between the northern and southern sites (Supplementary Fig. S1).

RRV outbreaks in semi-arid and tropical northern sites were positively predicted by weekly precipitation and minimum temperatures (Table 1). Weekly maximum tide heights were further positive predictors for outbreak probabilities in tropical Derby and 

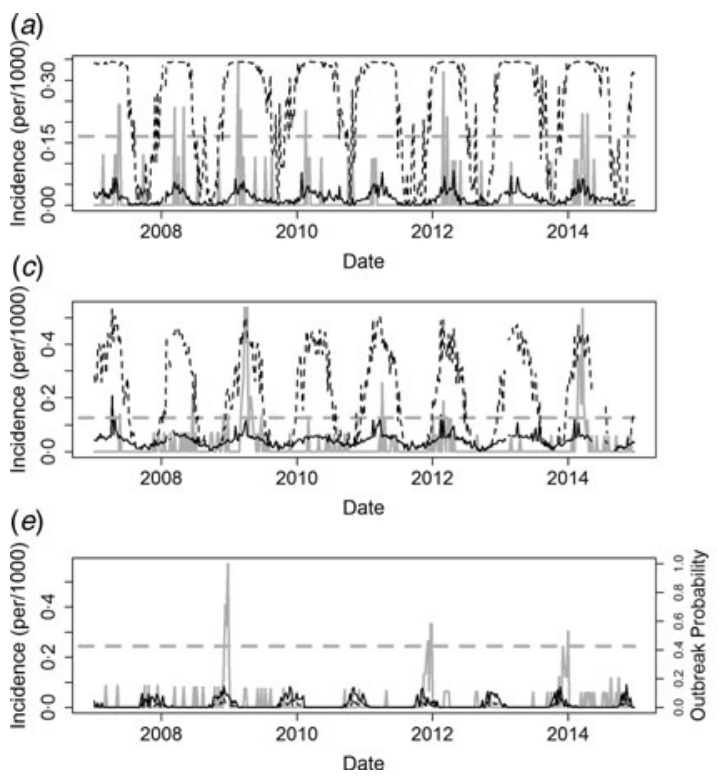

(b)

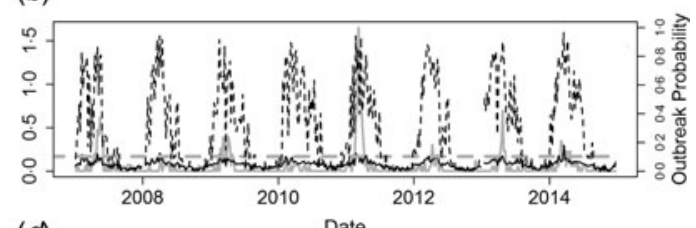

(d)

Date
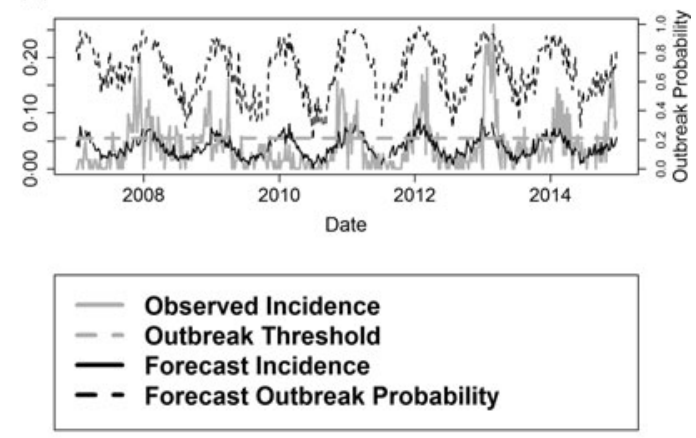

Fig. 2 Outbreak probabilities and incidence across all sites. Observed incidence (left axis) plotted against the threshold, which defines and outbreak (left axis), predicted outbreak probabilities (right axis), and predicted incidence (per/1000 individuals, left axis) for a 7-year timespan from 2007 to 2014 for five sites in Western Australia: a, Derby; b, Broome; c, Port Hedland; d, Mandurah; e, Capel.

Broome, with weekly maximum temperature also becoming an important predictor in Derby (Table 1). For the Southern Mediterranean climate site of Mandurah, in comparison, RRV outbreaks were predicted by weekly minimum and maximum temperatures and maximum tide heights (Table 1). With the exception of the semi-arid Port Hedland, maximum tide heights had similar importance in model selection between the northern and southern sites. The weekly lag between precipitation and RRV activity was greater in the southern sites, relative to the northern sites (Table 1). Variable importance suggested that weekly minimum temperatures in the northern sites had a greater relative influence on the probability of an outbreak than in the southern sites. Furthermore, weekly maximum temperature had a greater variable importance weight in the southern site of Mandurah than that of the northern sites (Table 1).

Outbreak predictions of observed RRV outbreaks made from the modelled averaged coefficients had high sensitivity (Fig. 2, Table 2). Specificity on the other hand was lower (Table 2). Models for the sites Broome and Port Hedland both had the greatest balance between high model sensitivity and specificity (Table 2). This was reflected when visually plotted, where weekly outbreak probabilities and predicted incidence increase when observed incidence rose (Fig. 2). The error from the $K$-fold cross-validation suggests that the models were consistently predicting outcomes of similar accuracy across the entire study period for all sites (Table 2). The exception to this was Capel, where outbreaks were not well predicted for.

\section{The weekly severity of an outbreak}

Similar to predicting outbreaks, weekly RRV incidence across the northern sites were driven by weekly minimum temperatures and precipitation (Table 3). There were some differences in predictors of RRV for the northern sites. For instance, weekly maximum temperatures and tidal heights were associated with RRV incidence in Derby, and maximum tide heights in Broome (Table 3). Weekly RRV incidence in the southern site of Mandurah was predicted by weekly minimum temperatures and maximum tide heights (Table 3). For Capel minimum temperatures exhibited a trending relationship to RRV incidence, and incidence was negatively associated with maximum temperatures.

While predicted incidence were generally conservative, prediction trends matched that of weekly observed incidence (Fig. 2). $K$-fold cross-validation of the linear regression models, with the exception of Capel, found very little variation in cross-validation error suggesting the model accuracy and agreement 
Table 1 Environmental hurdle model characteristics of Ross River virus across all sites.

\begin{tabular}{|c|c|c|c|c|c|}
\hline \multirow[b]{2}{*}{$\begin{array}{l}\text { Site \& } \\
\text { variable }\end{array}$} & \multirow[b]{2}{*}{$\begin{array}{l}\text { Lag } \\
\text { (weeks) }\end{array}$} & \multicolumn{4}{|c|}{ Hurdle Model } \\
\hline & & OR & $\begin{array}{l}\text { SE } \\
(\operatorname{logit})\end{array}$ & $\begin{array}{l}\text { Variable } \\
\text { importance }\end{array}$ & $P$-value \\
\hline \multicolumn{6}{|l|}{ Derby } \\
\hline$T_{\min } *$ & 10 & $1 \cdot 640$ & $0 \cdot 151$ & $0 \cdot 99$ & $0 \cdot 001$ \\
\hline$T_{\max } *$ & 18 & $1 \cdot 296$ & $0 \cdot 110$ & $0 \cdot 47$ & $0 \cdot 018$ \\
\hline $\mathrm{TH}_{\max } *$ & 2 & $1 \cdot 017$ & 0.003 & $0 \cdot 84$ & $<0.001$ \\
\hline$P_{\mathrm{tot}}^{*}$ & 6 & $1 \cdot 004$ & $0 \cdot 003$ & $0 \cdot 49$ & $0 \cdot 215$ \\
\hline \multicolumn{6}{|l|}{ Broome } \\
\hline$T_{\min } *$ & 13 & $1 \cdot 447$ & $0 \cdot 070$ & $>0.99$ & $<0 \cdot 001$ \\
\hline$T_{\max }$ & 18 & $1 \cdot 067$ & 0.075 & $0 \cdot 22$ & $>0.999$ \\
\hline $\mathrm{TH}_{\min }$ & 16 & 0.999 & $0 \cdot 002$ & $0 \cdot 09$ & 0.527 \\
\hline $\mathrm{TH}_{\max } *$ & 0 & $1 \cdot 012$ & $0 \cdot 004$ & $>0.99$ & $<0 \cdot 001$ \\
\hline$P_{\text {tot }}^{*}$ & 8 & $1 \cdot 011$ & $0 \cdot 002$ & $>0.99$ & $<0.001$ \\
\hline \multicolumn{6}{|c|}{ Port Hedland } \\
\hline$T_{\min } *$ & 10 & $1 \cdot 384$ & $0 \cdot 056$ & $>0.99$ & $<0.001$ \\
\hline$T_{\max }$ & 12 & $1 \cdot 057$ & $0 \cdot 062$ & $0 \cdot 16$ & $0 \cdot 372$ \\
\hline $\mathrm{TH}_{\min }$ & 8 & $1 \cdot 002$ & 0.003 & $0 \cdot 15$ & $0 \cdot 440$ \\
\hline $\mathrm{TH}_{\max }$ & 5 & $1 \cdot 002$ & $0 \cdot 004$ & $0 \cdot 12$ & 0.617 \\
\hline$P_{\text {tot }}{ }^{*}$ & 5 & $1 \cdot 012$ & 0.003 & $0 \cdot 98$ & $<0.001$ \\
\hline \multicolumn{6}{|l|}{ Mandurah } \\
\hline$T_{\min } *$ & 0 & $1 \cdot 166$ & 0.062 & $0 \cdot 64$ & $0 \cdot 014$ \\
\hline$T_{\max } *$ & 0 & $1 \cdot 115$ & 0.043 & $0 \cdot 64$ & $0 \cdot 011$ \\
\hline $\mathrm{TH}_{\min }$ & 6 & $1 \cdot 007$ & $0 \cdot 010$ & $0 \cdot 17$ & $0 \cdot 470$ \\
\hline $\mathrm{TH}_{\max } *$ & 0 & $1 \cdot 020$ & 0.007 & $0 \cdot 90$ & $0 \cdot 003$ \\
\hline$P_{\text {tot }}$ & 10 & $1 \cdot 004$ & $0 \cdot 007$ & $0 \cdot 13$ & 0.525 \\
\hline \multicolumn{6}{|l|}{ Capel } \\
\hline$T_{\min } *$ & 18 & 0.853 & $0 \cdot 080$ & $0 \cdot 47$ & $0 \cdot 488$ \\
\hline$T_{\max } *$ & 18 & 0.699 & $0 \cdot 129$ & $0 \cdot 90$ & 0.006 \\
\hline$P_{\text {tot }}$ & 11 & 1.009 & 0.009 & $0 \cdot 18$ & 0.297 \\
\hline
\end{tabular}

Environmental variables predicting RRV outbreaks: OR, odds ratio; SE, standard error. Where the values: $P_{\text {tot }}$, is for weekly precipitation; $T_{\min }$, minimum temperature; $T_{\max }$, maximum temperature; $\mathrm{TH}_{\min }$, minimum tide height; and $\mathrm{TH}_{\max }$, maximum tide height.

* Represents environmental variables included in the best-fit model.

in predictability when validated against the entire data set in their repeated predictability (Table 3 ).

\section{Timing of mitigation expenditure to RRV incidence}

To examine the relationship between RRV incidence and expenditure on mitigation in Mandurah from 2007 to 2014 (while taking into account the environmental determinates of incidence) we undertook a linear regression analysis on residual RRV incidence from the model-averaged full model. Residual RRV incidence was positively related to expenditure $\left(\beta^{\text {(cost) }}=7 \cdot 60 \times 10^{-06}, F=19 \cdot 7, \mathrm{df}=70, P=<0 \cdot 001\right)$, suggesting proportionality between the two (Fig. 3). Model accuracy assessed using a $K$-fold crossvalidation with 4-folds repeated ten times, showed model agreement indicated consistency in this relationship (overall cross-validation error $=0 \cdot 014$ ).

\section{Forecasting tool}

We utilised our weekly modelling of outbreaks and incidence to create a user-friendly forecasting tool for each site (Supplementary Table S8). Utilising the environmental lags, predictions into the future could be made. Across the sites, future predictions could be made for: 2 weeks into the future for Derby, 1 week into the future for Broome, 5 weeks into the future for Port Hedland, and 1 week into the future for Mandurah, Capel was omitted from the forecasting tool due to poor model performance.

\section{DISCUSSION}

Transmission of mosquito-borne disease is controlled by a combination of environmental, and vector and reservoir host dynamics, which vary across geographical and climatic regions. In forecasting the transmission of RRV, previous studies have used monthly environmental data and have highlighted the importance of having mosquito trapping data to supplement environmental predictors $[13,30,36]$. While these studies have been vital in developing our understanding of RRV epidemics, their course temporal resolution and recruitment for labour intensive surveillance [13, 24, 29, 37-42] limit practical application for timely forecasts to inform mitigation activities and public awareness campaigns.

Our weekly temporal scale forecast models suggest that in Western Australia, the environmental determinants of RRV activity differs among climatic regions. Southern Mediterranean regions are less dependent on precipitation compared with tropical and semi-arid environments in the northern areas of the state. Precipitation did not significantly predict RRV outbreaks or incidence in the Mediterranean sites compared to the semi-arid and tropical sites. The fine-temporal drivers of RRV across climatic regions (Mediterranean, semi-arid, and tropical) are poorly studied and critical in creating effective region-specific mosquito-borne disease management.

Differences in environmental drivers of RRV in this study may be reflective of geographical differences in landscape between these regions. The semi-arid and 
Table 2 Sensitivity, specificity, and cross-validation of model performance and predictions

\begin{tabular}{|c|c|c|c|c|c|}
\hline & Sensitivity & Specificity & PPV & NPV & $K$-fold cross-validation error (range) \\
\hline Derby & 1 & $0 \cdot 304$ & $0 \cdot 034$ & 1 & $0 \cdot 0224(0 \cdot 0221-0 \cdot 0225)$ \\
\hline Broome & $0 \cdot 798$ & $0 \cdot 831$ & $0 \cdot 263$ & $0 \cdot 982$ & $0.0545(0.0542-0 \cdot 0549)$ \\
\hline Port Hedland & $0 \cdot 914$ & $0 \cdot 656$ & $0 \cdot 123$ & $0 \cdot 993$ & $0 \cdot 0454(0 \cdot 0450-0 \cdot 0465)$ \\
\hline Mandurah & $0 \cdot 975$ & $0 \cdot 179$ & $0 \cdot 192$ & $0 \cdot 973$ & $0 \cdot 1814(0 \cdot 1801-0 \cdot 1824)$ \\
\hline Capel & 0 & 1 & NA & $0 \cdot 973$ & $0 \cdot 0463(0 \cdot 0459-0 \cdot 0466)$ \\
\hline
\end{tabular}

Sensitivity and specificity of the predictions produced from the hurdle models using a standard 0.5 cut-off point and the crossvalidation error. PPV and NPV represent positive and negative predictive values, respectively.

Table 3 Environmental linear regression model characteristics of Ross River virus across all sites

\begin{tabular}{|c|c|c|c|c|c|}
\hline & $\beta$ & $\mathrm{SE}$ & $\begin{array}{l}\text { Variable } \\
\text { importance }\end{array}$ & $P$-value & $\begin{array}{l}K \text {-fold cross-validation } \\
\text { error (range) }\end{array}$ \\
\hline \multicolumn{6}{|l|}{ Derby } \\
\hline$T_{\min }$ & $9 \cdot 302 \times 10^{-04}$ & $3.355 \times 10^{-04}$ & $0 \cdot 95$ & $0 \cdot 006$ & \multirow[t]{4}{*}{$0 \cdot 002(0 \cdot 001-0 \cdot 006)$} \\
\hline$T_{\max }$ & $1.603 \times 10^{-03}$ & $4.666 \times 10^{-04}$ & 0.99 & $<0 \cdot 001$ & \\
\hline $\mathrm{TH}_{\max }$ & $1 \cdot 153 \times 10^{-04}$ & $3 \cdot 311 \times 10^{-05}$ & 0.99 & $<0 \cdot 001$ & \\
\hline$P_{\text {tot }}$ & $2 \cdot 291 \times 10^{-04}$ & $3.904 \times 10^{-05}$ & $>0.99$ & $<0 \cdot 001$ & \\
\hline \multicolumn{6}{|l|}{ Broome } \\
\hline$T_{\min }$ & $4 \cdot 159 \times 10^{-03}$ & $6.535 \times 10^{-04}$ & $>0.99$ & $<0.001$ & \multirow[t]{5}{*}{$0 \cdot 010(0 \cdot 004-0 \cdot 018)$} \\
\hline$T_{\max }$ & $1.904 \times 10^{-03}$ & $1.343 \times 10^{-03}$ & $0 \cdot 50$ & $0 \cdot 157$ & \\
\hline $\mathrm{TH}_{\min }$ & $5 \cdot 495 \times 10^{-05}$ & $3.699 \times 10^{-05}$ & $0 \cdot 52$ & $0 \cdot 138$ & \\
\hline $\mathrm{TH}_{\max }$ & $2 \cdot 160 \times 10^{-04}$ & $5.034 \times 10^{-05}$ & $>0.99$ & $<0.001$ & \\
\hline$P_{\text {tot }}$ & $7 \cdot 036 \times 10^{-04}$ & $7 \cdot 850 \times 10^{-05}$ & $>0.99$ & $<0 \cdot 001$ & \\
\hline \multicolumn{6}{|c|}{ Port Hedland } \\
\hline$T_{\min }$ & $2.873 \times 10^{-3}$ & $4.567 \times 10^{-4}$ & $>0.99$ & $<0.001$ & \multirow[t]{5}{*}{$0 \cdot 004(0 \cdot 002-0 \cdot 007)$} \\
\hline$T_{\max }$ & $-2 \cdot 255 \times 10^{-4}$ & $7 \cdot 272 \times 10^{-4}$ & $0 \cdot 28$ & $0 \cdot 757$ & \\
\hline $\mathrm{TH}_{\min }$ & $4.063 \times 10^{-5}$ & $3 \cdot 911 \times 10^{-5}$ & $0 \cdot 38$ & $0 \cdot 309$ & \\
\hline $\mathrm{TH}_{\max }$ & $5.553 \times 10^{-5}$ & $4.890 \times 10^{-5}$ & $0 \cdot 41$ & $0 \cdot 257$ & \\
\hline$P_{\text {tot }}$ & $6 \cdot 136 \times 10^{-4}$ & $8.485 \times 10^{-8}$ & $>0.99$ & $<0 \cdot 001$ & \\
\hline \multicolumn{6}{|c|}{ Mandurah } \\
\hline$T_{\min }$ & $4 \cdot 027 \times 10^{-3}$ & $1.459 \times 10^{-3}$ & $0 \cdot 89$ & $0 \cdot 006$ & \multirow[t]{5}{*}{$0 \cdot 004(0 \cdot 001-0 \cdot 004)$} \\
\hline$T_{\max }$ & $1.800 \times 10^{-3}$ & $1 \cdot 204 \times 10^{-3}$ & $0 \cdot 55$ & $0 \cdot 135$ & \\
\hline $\mathrm{TH}_{\min }$ & $1.043 \times 10^{-4}$ & $2 \cdot 419 \times 10^{-4}$ & $0 \cdot 30$ & 0.667 & \\
\hline $\mathrm{TH}_{\max }$ & $5.676 \times 10^{-4}$ & $1.793 \times 10^{-4}$ & $0 \cdot 98$ & $0 \cdot 002$ & \\
\hline$P_{\text {tot }}$ & $2 \cdot 044 \times 10^{-4}$ & $1.689 \times 10^{-4}$ & $0 \cdot 43$ & $0 \cdot 227$ & \\
\hline \multicolumn{6}{|l|}{ Capel } \\
\hline$T_{\min }$ & $-5 \cdot 292 \times 10^{-3}$ & $3 \cdot 149 \times 10^{-3}$ & $0 \cdot 60$ & $0 \cdot 093$ & \multirow[t]{3}{*}{$0.033(0.003-0.047)$} \\
\hline$T_{\max }$ & $-1 \cdot 193 \times 10^{-2}$ & $3.925 \times 10^{-3}$ & $0 \cdot 97$ & $0 \cdot 002$ & \\
\hline$P_{\text {tot }}$ & $4 \cdot 166 \times 10^{-4}$ & $3.553 \times 10^{-4}$ & $0 \cdot 42$ & $0 \cdot 242$ & \\
\hline
\end{tabular}

Environmental variables predicting RRV incidence: $\beta$, beta coefficient; $\mathrm{SE}$, standard error; and the $K$-fold cross-validation error. Where the values: $P_{\text {tot }}$, is for weekly precipitation; $T_{\min }$, minimum temperature; $T_{\max }$, maximum temperature; $\mathrm{TH}_{\min }$, minimum tide height; and $\mathrm{TH}_{\max }$, maximum tide height.

tropical sites have extensive mangrove and saltmarsh areas surrounding them, becoming inundated by precipitation leading to mosquito breeding. While the Mediterranean site of Mandurah has an extensive waterway system resulting in a lower impact of precipitation on RRV transmission. Similar to previous studies in the south-western parts of Western Australia [13, 43] we found that in the Mediterranean site of Mandurah, weekly maximum and minimum temperatures and maximum tide heights were important contributors to RRV epidemics. In the semi-arid and tropical sites the weekly environmental factors minimum temperature, maximum tide height, and precipitation generally predicted RRV notifications and transmission with 


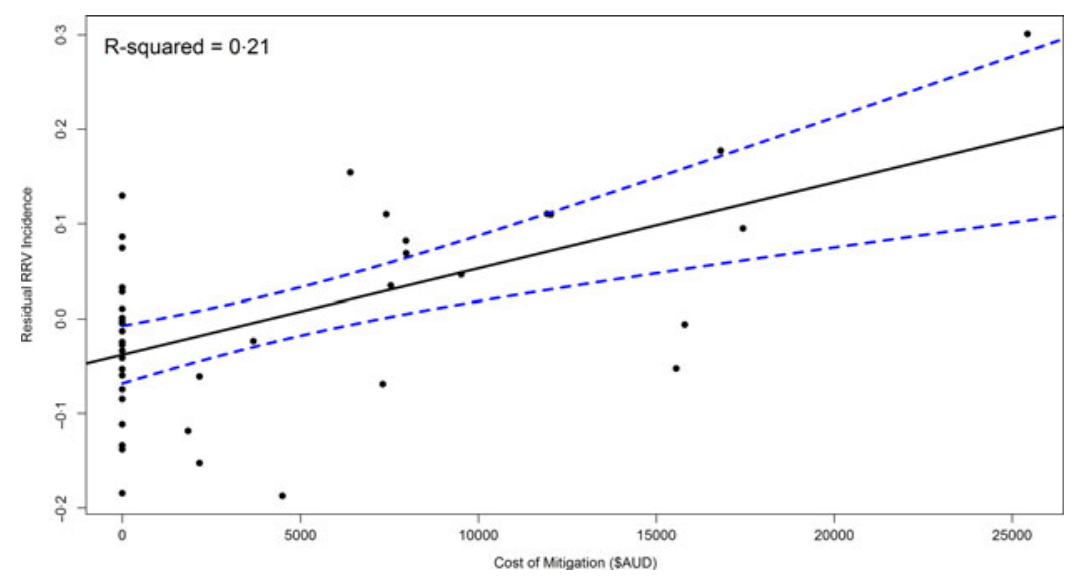

Fig. 3 Mitigation expenditure on residual Ross River virus (RRV) incidence. Mitigation expenditure influence on RRV incidence for Mandurah. The best-fit model combination residuals of a linear model being predicted by the cost of mitigation on a monthly basis $(95 \%$ confidence intervals in blue).

minor differences between these regions. This may demonstrate the role geographical and environmental characteristics have among Mediterranean, semi-arid, and tropical regions. The models constructed for Mandurah were the only ones to consistently have predicted incidence exceeding the threshold that we classified as an outbreak of RRV. The remaining sites' predictions may be conservative due to the relative small human populations and low cases of RRV being reported. While incidence prediction may be conservative for Derby, Broome, and Port Hedland, periods of sustained RRV activity and peaks still provide useful information during decision-making processes when undertaking mitigation activities.

This research also supports previous studies on mosquito-host interactions, their role in RRV transmission and outbreaks, owing to maximum tidal heights in estuarine, flood plans, and wetland areas [42]. The tropical regions of Derby and Broome experience some of the highest tidal variation in Australia, and in the southern hemisphere [44, 45]. In both Derby and Broome the weekly maximum tidal height was an important driver in the probability of an outbreak and also incidence, with the inundation of mangroves being known to force animal reservoirs closer to urban areas [16]. The Southern Mediterranean site of Mandurah has an extensive tidal estuarine salt marsh area coupled with inflowing rivers creating ideal conditions for mosquito breeding $[46,47]$. In Mandurah, weekly maximum tide height had similar odds of increasing the probability of an outbreak as the tropical sites. However in Port Hedland, RRV outbreaks were not significantly predicted by maximum tide height. Instead, precipitation in Port Hedland had a greater influence on the odds of a RRV outbreak than that of any other study site. Port Hedland, in contrast to our other coastal sites, is industrialised and frequently used as a major shipping transport hub, has three surrounding suburbs, which are further inland, and has a deeper port within the estuary.

Following a precipitation event in coastal areas, the northern tropical regions of Western Australia experienced RRV activity sooner than that of Mediterranean southern regions. However, the southern site of Mandurah experienced much shorter environmental lags in RRV activity from changes in temperature than that of the northern sites. Both these findings may reflect the environmental differences among Mediterranean, semi-arid, and tropical regions and are of importance when tackling mosquito-borne diseases at a regional level. These lagged effects of RRV activity occurring after an environmental event vary greatly between regions across Australia, reflecting the biotic and abiotic processes involved in the transmission [5, 10, 16, 24, 48]. Our findings help to illustrate the latitudinal responses in RRV activity in relation to environmental factors among climates [49].

While the economic impact of RRV infections have been investigated [50, 51], there is often little knowledge of the role mitigation expenditure has in association with RRV activity [50, 51]. Our findings indicate that while variable, expenditure increases proportional to that of residual RRV notifications (after having controlled for the effect of environmental predictors). This finding helps to support and justify current mitigation practices already in place, and variability in the association illustrates the complex nature of managing 
this mosquito-borne disease. It could be argued that the relationship between mitigation expenditure and RRV incidence should be negative. However, it is important to recognise that mosquito mitigation is only carried out during times when mosquito control officers suspect mosquito breeding is most likely, and potential for disease outbreak is greater. Thus, when RRV incidence is likely to be at its greatest, so too should mitigation expenditure. As a consequence, the strength of the positive association becomes a proxy for the correct timing for mitigation activities and expenditure.

A considerable strength to this study is the extensive history of RRV notifications and environmental data in both constructing forecasts and validating the models. This is the first study, to our knowledge, to employ hurdle models to predict longitudinal RRV outbreak dynamics [52]. While our approach does not necessarily imply our models are superior to previous methods, our models do allow for alternative approaches when modelling mosquito-borne disease data at fine-temporal resolutions. Previous studies forecasting RRV outbreaks and their associated predictors have used logistic and negative binomial regression, and seasonal autoregressive integrated moving average (SARIMA) modelling techniques [5, 13, 14, 24, 49]. Hurdle models offer some distinct attributes that may make them well suited to predicting outbreaks. From our models, we found strong agreement among our model validation analyse. Model sensitivity and specificity in predicting outbreaks were generally greater than other studies, and these sensitivities rivalled those that explicitly include mosquito surveillance in modelling [13].

Resulting from our findings was the construction of early warning forecasting tools for RRV outbreaks and incidence among our study sites. Previous epidemiological studies of RRV often suggest that early warning forecasting systems can be developed and would be of benefit for public health and mosquito control programmes [13, 31, 48]. Yet, to our knowledge, very few studies take the much needed step of translating their findings into a user friendly tool. Here we have applied our findings into a practical user friendly forecasting tool, which has been delivered to the Western Australian department of health and local councils, and is available in the supplementary material to this article.

It is notable that for our study site of Capel, the models predicting outbreaks and incidence were poor fits to the RRV notifications comparative to that of the other sites. One reason for this could be the source of temperature data, which were unavailable for Capel and had to be gathered from a neighbouring town. Furthermore, the dynamics of RRV notifications at Capel were quite sporadic, and the low population size of the town meant that high sporadic numbers of RRV notifications had large influences of levels of incidence. These limitations are unfortunately difficult to overcome at this location, and accordingly we are tentative in our interpretations of results from this site. Moreover, the variation of mosquito-control programmes and its relative effect to RRV notifications was not assessed here and would provide valuable insight into the role it plays in relation to environmental conditions and RRV outbreaks.

\section{CONCLUSION}

We present models capable of filling the void in forecasting RRV outbreaks and disease incidence on a weekly temporal scale and across multiple climates. The use of these models is particularly valuable for forecasting RRV without the need for mosquito surveillance. We also demonstrate the complex nature of RRV transmission between climatically difference regions, and the necessity to understand these differences when creating effective forecasting tools. Forecasting weekly RRV outbreaks and incidence may help with timing of mitigation activities and public health notifications at critical times when disease risk is greatest. The early warning predictive tool developed here helps translate epidemiological modelling studies into easy to use tools that can be adopted by regional and local health bodies, which may inform local decision maker's processes. The coupling of the predictive modelling with mitigation expenditure is also informative of the extent to which mitigation activities are well timed with RRV incidence. While this study bridges previous knowledge gaps in fine-temporal transmission dynamics of RRV, and the role mitigation has during outbreaks, we highlight the need for further research extending these analyses across multiple sites in similar climatic regions, so that broader generalisations may be made to assist the public health and vector management community.

\section{SUPPLEMENTARY MATERIAL}

The supplementary material for this article can be found at https://doi.org/10.1017/S095026881700190X 


\section{ACKNOWLEDGEMENTS}

The authors would like to thank Peter Neville and the Western Australia Department of Health for access to the notification data and for the 'on the ground' aspect of this study. Financial support for this project was provided through the Funding Initiative for Mosquito Management In Western Australia Research Grants, administered by the Department of Health, Western Australia.

\section{REFERENCES}

1. Gratz NG. Emerging and resurging vector-borne diseases. Annual Review of Entomology 1999; 44: 51-75.

2. Mackenzie JS, Gubler DJ, Petersen LR. Emerging flaviviruses: the spread and resurgence of Japanese encephalitis, West Nile and dengue viruses. Nature Medicine 2004; 10: 98-109.

3. Tipayamongkholgul M, et al. Effects of the El NinoSouthern Oscillation on dengue epidemics in Thailand, 1996-2005. BioMed Central Public Health. 2009; 9: 1-15.

4. Naish S, Hu W, et al. Weather variability, tides, and barmah forest virus disease in the gladstone region, Australia. Environmental Health Perspectives 2006; 114: 678-683.

5. Yu W, Dale $\mathbf{P}$, Turner $\mathbf{L}$, Tong $\mathbf{S}$. Projecting the impact of climate change on the transmission of Ross River virus: methodological challenges and research needs. Epidemiology and Infection 2014; 142: 2013-2023.

6. Harley D, Sleigh A, Ritchie S. Ross River virus transmission, infection, and disease: a cross-disciplinary review. Clinical Microbiology Reviews 2001; 14: 909-932.

7. Aaskov J, Fokine A, Liu W. Ross River virus evolution: implications for vaccine development. Future Virology 2012; 7: 173-178.

8. Australian Government Department of Health. Notifications for all diseases by State \& Territory and year (http://www9.health.gov.au/cda/source/rpt_2_sel. cfm). Accessed 16 September 2016.

9. Kelly-Hope LA, Purdie DM, Kay BH. Ross River virus disease in Australia, 1886-1998, with analysis of risk factors associated with outbreaks. Journal of Medical Entomology 2004; 41: 133-150.

10. Russell RC. Ross river virus: ecology and distribution. Annual Review of Entomology 2002; 47: 1-31.

11. Carver S, et al. Influence of hosts on the ecology of arboviral transmission: potential mechanisms influencing dengue, Murray Valley encephalitis, and Ross River virus in Australia. Vector-Borne and Zoonotic Diseases 2009; 9: 51-64.

12. Claflin SB, Webb CE. Ross River virus: many vectors and unusual hosts make for an unpredictable pathogen. PLoS Pathogens 2015; 11: 1-5.

13. Woodruff RE, et al. Early warning of Ross River virus epidemics - combining surveillance data on climate and mosquitoes. Epidemiology 2006; 17: 569-575.
14. Tong S, et al. Climate variability, social and environmental factors, and Ross River virus transmission: research development and future research needs. Environmental Health Perspectives 2008; 116: 15911597.

15. Tall JA, Gatton ML, Tong SL. Ross River virus disease activity associated with naturally occurring nontidal flood events in Australia: a systematic review. Journal of Medical Entomology 2014; 51: 1097-1108.

16. Jacups SP, Whelan PI, Currie BJ. Ross River virus and Barmah Forest virus infections: a review of history, ecology, and predictive models, with implications for tropical northern Australia. Vector-Borne and Zoonotic Diseases 2008; 8: 283-297.

17. Haynes CD, Ridpath MG, Williams MAJ. Monsoonal Australia: Landscape, Ecology and Man in the Northern Lowlands. Rotterdam: A.A. Balkema, 1991.

18. Carver S, et al. Environmental monitoring to enhance comprehension and control of infectious diseases. Journal of Environmental Monitoring 2010; 12: 2048 2055.

19. Woodruff RE, et al. Predicting Ross River virus epidemics from regional weather data. Epidemiology 2002; 13: 384-393.

20. Russell RC. Seasonal activity and abundance of the arbovirus vector Culex annulirostris skuse near echuca, Victoria, in the Murray Valley of southeastern Australia 1979-1985. Australian Journal of Experimental Biology \& Medical Science 1986; 64: 97-103.

21. Rowbottom R, et al. Resource limitation, controphic ostracod density and larval mosquito development. PLOS ONE 2015; 10: 1-13.

22. Lindsay M, et al. Ross River and Barmah Forest viruses in Western Australia, 2000/01-2003/04: contrasting patterns of disease activity. Arbovirus Research in Australia 2005; 9: 194-201.

23. Lindsay M, et al. Western Australian arbovirus surveillance and research program, Annual Report: 1997-1998. Arbovirus Surveillance and Research Laboratory and the Western Australian Centre for Pathology and Medical Research. Western Australian Government, 1998.

24. McIver L, et al. A climate-based early warning system to predict outbreaks of Ross River virus disease in the Broome region of western Australia. Australian and New Zealand Journal of Public Health 2010; 34: 89-90.

25. Severn S, Brendan I. 2013/14 Mosquito Management Annual Report. Mandurah, 2014.

26. Australia Bureau of Statistics. 3218.0 - Regional Population Growth, Australia, 2013-14 (http://www.abs. gov.au/AUSSTATS/abs@.nsf/DetailsPage/3218.0201314? OpenDocument). Accessed 2 February 2016.

27. Brady OJ, et al. Dengue disease outbreak definitions are implicitly variable. Epidemics 2015; 11: 92-102.

28. Abraham B, Ledolter J. Statistical Methods for Forecasting. New York: Wiley, 1983.

29. Tong S, Hu W. Different responses of Ross River virus to climate variability between coastline and inland cities in Queensland, Australia. Occupational and Environmental Medicine 2002; 59: 739-744. 
30. Jacups SP, et al. Determining meteorological drivers of salt marsh mosquito peaks in tropical northern Australia. Journal of Vector Ecology 2015; 40: 277-281.

31. Williams CR, Fricker SR, Kokkinn MJ. Environmental and entomological factors determining Ross River virus activity in the River Murray Valley of South Australia. Australian and New Zealand Journal of Public Health 2009; 33: 284-288.

32. Burnham KP, Anderson DR. Model Selection and Multimodel Inference : A Practical Information-Theoretic Approach. New York: Springer, 2002.

33. Wong TT. Performance evaluation of classification algorithms by k-fold and leave-one-out cross validation. Pattern Recognition. 2015; 48: 2839-2846.

34. Meijer RJ, Goeman JJ. Efficient approximate k-fold and leave-one-out cross-validation for ridge regression. Biometrical Journal 2013; 55: 141-155.

35. Rodriguez JD, Perez A, Lozano JA. Sensitivity analysis of $\mathrm{k}$-fold cross validation in prediction error estimation. IEEE Transactions on Pattern Analysis and Machine Intelligence 2010; 32: 569-575.

36. Jacups SP, et al. Predictive indicators for Ross River virus infection in the Darwin area of tropical northern Australia, using long-term mosquito trapping data. Tropical Medicine \& International Health 2008; 13: 943-952.

37. Bi P, Parton KA. Climate variations and the transmission of Ross River virus infection in coastal and inland region of Queensland: an analysis from Townsville and Toowoomba. Environmental Health 2003; 3: 73-80.

38. Hu W, et al. Difference in mosquito species (Diptera: Culicidae) and the transmission of Ross River virus between coastline and inland areas in Brisbane, Australia. Environmental Entomology 2010; 39: 88-97.

39. Hu W, et al. Development of a predictive model for Ross river virus disease in Brisbane, Australia. The American Journal of Tropical Medicine and Hygiene 2004; 71: 129-137.

40. Hu W, et al. Mosquito species (Diptera: Culicidae) and the transmission of Ross River virus in Brisbane, Australia. Journal of Medical Entomology 2006; 43: 375-381.
41. Hu WB, et al. Rainfall, mosquito density and the transmission of Ross River virus: a time-series forecasting model. Ecological Modelling 2006; 196: 505-514.

42. Tong $\mathbf{S}$, et al. Climatic, high tide and vector variables and the transmission of Ross River virus. Internal Medicine Journal 2005; 35: 677-680.

43. Mackenzie JS, et al. Arboviruses causing humandisease in the Australasian zoogeographic region. Archives of Virology 1994; 136: 447-467.

44. Wright AE, et al. A preliminary investigation of the ecology of arboviruses in the derby area of the Kimberley region, western Australia. Australian Journal of Experimental Biology and Medical Science 1981; 59: 357-367.

45. Shire of Derby Council. Shire of Derby/West Kimberly: Shire of Derby (http://www.sdwk.wa.gov.au/). Accessed 5 May 2016.

46. Jardine A, Neville PJ, Lindsay MD. Proximity to mosquito breeding habitat and Ross River virus risk in the Peel region of Western Australia. Vector-Borne and Zoonotic Diseases 2015; 15: 141-146.

47. Lindsay M, et al. An outbreak of Ross River virus disease in the south-west of Western Australia. Communicable Diseases Intelligence 1996; 20: 136-139.

48. Werner AK, et al. Environmental drivers of Ross River virus in southeastern Tasmania, Australia: towards strengthening public health interventions. Epidemiology and Infection. 2012; 140: 359-371.

49. Gatton ML, Kay BH, Ryan PA. Environmental predictors of Ross River virus disease outbreaks in Queensland, Australia. The American Journal of Tropical Medicine and Hygiene 2005; 72: 792-799.

50. Geelhoed E. The Economic Costs of Ross River Virus Infection. Western Australia: Health Department of Western Australia, 1995.

51. Ratnayake JTB. The Valuation of Social and Economic Costs of Mosquito-transmitted Ross River Virus (Dissertation). Nathan, Queensland: Griffith University, 2005.

52. Mullahy J. Specification and testing of some modified count data models. Journal of Econometrics 1986; 33: 341-365. 